\title{
Desempenho funcional de crianças com paralisia cerebral de níveis socioeconômicos alto e baixo
}

\author{
Functional performance of children with cerebral palsy from high and low socioeconomic status
}

\author{
Elisângela Andrade Assis-Madeira1', Sueli Galego Carvalho², Silvana Maria Blascovi-Assis ${ }^{3}$
}

\section{RESUMO}

Objetivo: Investigar a influência do nível socioeconômico sobre o desempenho funcional de crianças com paralisia cerebral.

Métodos: Estudo transversal com abordagem quantitativa. Foram selecionadas 49 crianças com diagnóstico clínico de paralisia cerebral de uma amostra de conveniência, com idades de três a sete anos e meio, de ambos os sexos, classificadas de acordo com o nível de gravidade da paralisia cerebral, com base no Sistema de Classificação da Função Motora Grosseira. Os participantes foram distribuídos em dois grupos de níveis socioeconômicos, alto e baixo, de acordo com o Critério de Classificação Econômica do Brasil. O desempenho funcional foi avaliado com o Inventário de Avaliação Pediátrica de Incapacidade. Foi aplicado o teste $t$ de Student para amostras independentes a fim de comparar as médias entre os grupos.

Resultados: O nível socioeconômico não afetou o desempenho funcional de crianças com paralisia cerebral leve. Crianças com paralisia cerebral moderada de classe econômica baixa apresentaram escores da função social inferiores $(p=0,027)$ aos daquelas de classe alta. Crianças com paralisia cerebral grave de nível socioeconômico baixo apresentaram desempenho inferior nas habilidades de autocuidado $(p=0,021)$ e mobilidade $(p=0,005)$. Essas crianças foram mais dependentes em relação à mobilidade $(p=0,015)$ do que as de nível socioeconômico alto.

Conclusões: O nível socioeconômico pode exercer influência no desenvolvimento da criança com paralisia cerebral, devendo ser fator de risco considerado nas ações educacionais e de saúde voltadas a essa população.

Palavras-chave: paralisia cerebral; classe social; desenvolvimento infantil; criança.

\section{ABSTRACT}

Objective: To investigate the influence of socioeconomic status on the functional performance of children with cerebral palsy.

Methods: Cross-sectional quantitative study of 49 children diagnosed with cerebral palsy from a convenience sample. Children of both genders aged three to seven and a half years were studied. They were classified according to the level of severity of cerebral palsy based on the Gross Motor Function Classification System. Participants were organized in two groups considering their high or low socioeconomic status, according to the Brazilian Economic Classification Criteria. Functional performance was assessed by the Pediatric Evaluation of Disability Inventory. The Student's $t$-test was applied for independent samples in order to compare means between groups.

Results: Socioeconomic status did not affect functional performance of children with mild cerebral palsy. Those with moderate cerebral palsy and low socioeconomic status presented lower social function scores $(p=0.027)$ than those with high socioeconomic status. Children with severe cerebral palsy with low socioeconomic status presented worse performance in self-care skills $(p=0.021)$
Instituição: Universidade Presbiteriana Mackenzie, São Paulo, SP, Brasil ${ }^{1}$ Mestre em Distúrbios do Desenvolvimento pela Universidade Presbiteriana Mackenzie; Docente da Faculdade de Tecnologia e Ciências, Vitória da Conquista, BA, Brasil

2Doutora em Administração de Empresas pela Universidade Presbiteriana Mackenzie; Docente do Programa de Pós-graduação em Distúrbios do Desenvolvimento da Universidade Presbiteriana Mackenzie, São Paulo, SP, Brasil

${ }^{3}$ Doutora em Educação Física pela Universidade Estadual de Campinas (Unicamp); Docente do Programa de Pós-graduação em Distúrbios do Desenvolvimento da Universidade Presbiteriana Mackenzie, São Paulo, SP, Brasil

\section{Endereço para correspondência: \\ Elisângela Andrade Assis Madeira \\ Rua Pastor Valdomiro Oliveira, 737 - Candeias \\ CEP 45028-742 - Vitória da Conquista/BA \\ E-mail: eli.madeira@yahoo.com.br}

Fonte financiadora: Coordenação de Aperfeiçoamento de Pessoal de Nível Superior (Capes) e Fundo Mackenzie de Pesquisa (MackPesquisa)

Conflito de interesse: nada a declarar

Recebido em: 8/5/2012

Aprovado em: 24/9/2012 
and mobility $(p=0.005)$. These children were more dependent regarding mobility $(p=0.015)$ than those with high socioeconomic status.

Conclusions: Socioeconomic status may influence the development process of children with cerebral palsy and must be considered as a risk factor in educational and health practices aimed at this population.

Key-words: cerebral palsy; social class; child development; child.

\section{Introdução}

O desenvolvimento da criança é composto por vários domínios interdependentes (sensório-motor, cognitivo e socioemocional), que podem sofrer influência de fatores biológicos (idade gestacional e peso ao nascimento), socioambientais (condição econômica e escolaridade dos pais) e hereditários, sendo afetados por situações adversas ou favoráveis ${ }^{(1)}$. Quando tais fatores trazem consequências negativas ao desenvolvimento da criança, são chamados de risco. Dentre esses estão características familiares, as quais podem colocar em maior risco o desenvolvimento saudável da criança ${ }^{(2,3)}$.

O desenvolvimento motor é um processo de mudança no comportamento, resultado da interação da hereditariedade com o ambiente. Nesse processo, considera-se que o ambiente provoca um efeito estimulador que interage com a biologia humana, produzindo o comportamento ${ }^{(4)}$. Para Barros et al $l^{(5)}$, a identificação precoce de possíveis situações de risco que possam prejudicar o curso do desenvolvimento é importante para evitar danos ao longo da vida.

Algumas pesquisas evidenciam a relação entre baixa condição socioeconômica e comprometimento do desenvolvimento de crianças ${ }^{(1,6-8)}$. Elas apontam que o baixo nível socioeconômico (NSE) pode aumentar a vulnerabilidade biológica da criança, induzindo a resultados desfavoráveis no desenvolvimento. Infelizmente, a população que acumula fatores de risco que podem afetar o desenvolvimento é, quase sempre, a mais desfavorecida, aumentando, portanto, as chances de atraso.

A escolaridade do cuidador e a renda familiar são elementos básicos para determinar a saúde da criança, por serem indicadores de recursos disponíveis e conhecimento ou comportamento em relação à saúde e ao bem-estar da criança ${ }^{(9)}$.

O NSE pode ter impacto em variáveis do bem-estar de crianças com paralisia cerebral (PC), com distinção entre níveis altos e baixos de classes econômicas em relação às habilidades funcionais e independência. De acordo com Schenker $e t a l^{(10)}$, o desenvolvimento da motricidade da criança com PC apresenta caracteristicamente um atraso. Por isso, as tarefas motoras básicas podem ser adquiridas mais tardiamente na infância, gerando diferença de anos de atraso, quando comparadas com crianças hígidas no que diz respeito à motricidade grosseira. Nesse sentido, as manifestações funcionais das crianças com PC devem ser avaliadas, uma vez que o desempenho funcional sofre influência não exclusivamente das propriedades intrínsecas da criança, mas também das demandas específicas da tarefa e das características do ambiente no qual ela interage (11,12). $^{(1)}$

A relação entre NSE e PC ainda não é bem clara, porém, acredita-se que tal associação pode sugerir fatores etiológicos e caminhos para a prevenção ${ }^{(13)}$, bem como agravos ao desenvolvimento motor. $\mathrm{O}$ conhecimento do desempenho motor da PC, a partir de análise do contexto socioeconômico, pode representar um instrumento importante para tomadas de decisão na gestão de recursos e no planejamento de serviços voltados a essas crianças. Com base na literatura consultada, o presente estudo teve como objetivo investigar as possíveis relações entre o desempenho funcional de crianças com PC com diferentes graus de comprometimento e o NSE familiar.

\section{Método}

Foi realizado um estudo descritivo de corte transversal em instituições públicas e privadas da região metropolitana do Vale do Aço, no estado de Minas Gerais. O projeto de pesquisa foi previamente aprovado pelo Comitê de Ética em Pesquisa da Universidade Presbiteriana Mackenzie, atendendo às exigências da Resolução 196/96 do Conselho Nacional de Saúde. O termo de consentimento livre e esclarecido foi obtido do responsável legal dos participantes, após aceitarem voluntariamente participar do estudo. A coleta de dados foi realizada na instituição/clínica onde a criança era atendida, em sala reservada.

Foram incluídas na pesquisa crianças com diagnóstico clínico de PC de qualquer tipo e encefalopatia crônica não evolutiva (ECNE) de ambos os sexos, com idade cronológica de três a sete anos e meio; classificadas nos níveis I, II, III, IV e V no Sistema de Classificação da Função Motora Grosseira. No caso de crianças em uso de anticonvulsivantes, foram incluídas aquelas sem crises há pelo menos três meses. Foram excluídos sujeitos com malformação congênita não relacionada à PC, déficit sensorial grave, doenças cromossômicas, crianças submetidas à cirurgia do sistema musculoesquelético 
e/ou à aplicação de toxina botulínica do tipo A em tempo inferior a seis meses.

Participou deste estudo o principal cuidador da criança, responsável pelo bem-estar, assistência e prestação de cuidados; teria que residir ou passar a maior parte do dia com a criança (pelo menos meio período) e prestar assistência diária há pelo menos seis meses. Não foi realizado o cálculo amostral, pois o intuito foi coletar dados de crianças com PC que se encontrassem em reabilitação em instituições públicas e privadas e que preenchessem os critérios de inclusão para participar da pesquisa. Sendo assim, utilizou-se amostra de conveniência e foram selecionadas 49 crianças com PC.

A ficha de identificação incluiu dados da criança (idade, data e peso de nascimento, nomes do pai e da mãe, tempo de gestação, presença de complicações neonatais e epilepsia, se frequenta a escola) e do cuidador (idade, grau de parentesco com a criança, endereço, telefone, estado civil, nível escolar e ocupação). As crianças foram classificadas sempre pelo mesmo avaliador de acordo com o nível de gravidade da PC, com base no GMFCS $^{(14-16)}$. Tal instrumento estratifica as crianças com PC em níveis I, II, III, IV e V. Os pacientes foram agrupados em três níveis de comprometimento, de acordo com estudos que utilizaram igualmente esses sistemas ${ }^{(16,17)}$. As crianças classificadas nos níveis I e II foram consideradas como leves; as do nível III, como moderadas; e aquelas nos níveis IV e V, como graves. Os participantes também foram divididos em dois grupos de acordo com a classe econômica da família avaliada (alta e baixa), a partir do Critério de Classificação Econômica do Brasil ${ }^{(18)}$. As categorias socioeconômicas variam de A (muito alto) a $\mathrm{E}$ (muito baixo), sendo as demais categorias $(\mathrm{B}, \mathrm{C}, \mathrm{D})$ intermediárias. Para o estudo considerou-se que as categorias $\mathrm{A}$ e $\mathrm{B}$ pertenciam ao NSE alto, e as categorias C, D e E, ao baixo, conforme a classificação adotada por Mancini et $a l^{(19)}$. A distribuição final dos grupos ocorreu da seguinte forma: crianças de NSE alto (PC leve, moderada e grave) e crianças de NSE baixo (PC leve, moderada e PC grave).

Foi aplicada a versão brasileira do Inventário de Avaliação Pediátrica de Incapacidade (Pediatric Evaluation of Disability Inventory - PEDI) para analisar o desempenho funcional. Este teste é dividido em três partes distintas, que informam sobre três áreas de desempenho funcional. Neste estudo foram utilizadas somente as partes I e II; a terceira parte, de modificações do ambiente, não foi realizada. A primeira parte documenta as habilidades funcionais da criança nas seguintes escalas: autocuidado, mobilidade e função social. Cada item recebe escore 1 se a criança for capaz de desempenhar a atividade, ou 0 , se não for capaz de desempenhá-la. O total dos escores obtidos em cada escala dessa parte tem como resultado um escore total bruto para cada uma das três áreas de habilidades funcionais. A segunda parte do PEDI quantifica a ajuda fornecida pelo cuidador para a criança cumprir tarefas de: autocuidado, mobilidade e função social. Nesta parte do teste, a assistência é avaliada em escala ordinal, incluindo as categorias: 0 (assistência total), 1 (assistência máxima), 2 (assistência moderada), 3 (assistência mínima), 4 (supervisão) e 5 (independente). O manual do teste fornece critérios específicos para pontuar cada item. Neste estudo foram utilizados os dados brutos do $\mathrm{PEDI}^{(20)}$.

Os dados foram analisados empregando-se estatística descritiva e inferencial. Para realizar a análise estatística foi utilizado o software Statistical Package for the Social Sciences for Windows (SPSS), versão 11.0. As variáveis quantitativas foram descritas por meio de média e desvio padrão. Para verificar a normalidade dos dados, foi aplicado o teste de Kolmogorov-Smirnov, sendo aceita esta suposição: $p>0,05$. Já as variáveis qualitativas foram apresentadas por meio de frequências absolutas e relativas. A comparação dos grupos (gravidade da PC leve, moderada e grave) foram realizadas por análise de variância (ANOVA) para variáveis quantitativas (idade) e por qui-quadrado para as categóricas (sexo e NSE). A análise multivariada foi utilizada para verificar os efeitos de interação entre o NSE e a gravidade da PC no desempenho funcional (três gravidades de PC com dois NSEs), considerando-se um alfa de $5 \%$. Foi aplicado o teste $t$ para amostras independentes para comparar as médias entre os grupos. O nível de significância adotado foi de $5 \%$.

\section{Resultados}

As 49 crianças foram distribuídas de acordo com a classe social e a gravidade da PC em seis grupos distintos: Grupo $1(\mathrm{n}=8)$, para aquelas que tinham PC leve pertencentes a famílias das classes socioeconômicas A e B (NSE alto); Grupo $2(n=8), P C$ leve de famílias que pertencem às classes $C, D$ e E (NSE baixo); Grupo 3 ( $\mathrm{n}=7$ ), PC moderada pertencentes a famílias das classes socioeconômicas A e B (NSE alto); Grupo 4 ( $\mathrm{n}=10)$, PC moderada com famílias das classes C, D e E (NSE baixo); Grupo 5 ( $n=5)$, PC grave pertencentes a famílias das classes A e B (NSE alto); e Grupo 6 (n=11), PC grave com famílias das classes socioeconômicas C, D e E (NSE baixo). Foi possível notar que o Grupo 5 teve um número reduzido de crianças, o que se deveu ao fato de haver poucas crianças com PC grave de NSE alto e que preenchessem os critérios para participação neste estudo. 
A Tabela 1 apresenta informações descritivas das crianças, tais como: idade, sexo, NSE da família, idade e escolaridade do cuidador. No total, 20 crianças pertenciam ao NSE alto e 29 crianças pertenciam ao baixo.

$\mathrm{Na}$ análise multivariada não houve efeito de interação entre gravidade da PC e NSE $(p=0,141)$. Foi utilizado o teste $t$ para comparar o desempenho funcional das crianças pesquisadas entre as classes econômicas alta e baixa. Em relação às habilidades funcionais de crianças com PC leve (Tabela 2), foram comparadas as classes econômicas alta e baixa, porém, não foram evidenciadas diferenças significativas entre esses grupos. Observou-se que os grupos apresentaram desempenhos equivalentes na assistência do cuidador no autocuidado $(p=0,392)$, na mobilidade $(p=0,788)$ e na função social $(p=0,167)$, ou seja, não foi observada diferença significativa em tais áreas de função de crianças de ambos os NSE.

As habilidades funcionais de crianças com PC moderada também foram equivalentes nos NSE alto e baixo. Entretanto, na assistência do cuidador, os participantes de classe econômica baixa apresentaram escores da função social inferiores aos daqueles de classe alta $(p=0,027)$, ou seja, as crianças de NSE alto mostraram maior independência na função social do que aquelas de NSE baixo.

Tabela 1 - Análise descritiva dos grupos de gravidade das crianças com paralisia cerebral

\begin{tabular}{lcccc}
\hline Variáveis & \multicolumn{2}{c}{ Grupos } & \multirow{2}{*}{ Valor $\boldsymbol{p}$} \\
\cline { 2 - 3 } & Leve & Moderado & Grave & \multirow{2}{*}{0,037} \\
$\begin{array}{l}\text { Idade (anos) } \\
\text { Média } \pm \mathrm{DP}\end{array}$ & $5,1 \pm 1,5$ & $5,31 \pm 1,4$ & $5,0 \pm 1,4$ & \\
$\begin{array}{l}\text { Sexo } \\
\text { Masculino }\end{array}$ & 7 & 8 & 9 & 0,886 \\
$\quad$ Feminino & 9 & 9 & 7 & \\
NSE & 8 & 7 & 5 & 0,199 \\
$\quad$ Alto & 8 & 10 & 11 & \\
$\quad$ Baixo & $30,4 \pm 6,7$ & $33,4 \pm 6,8$ & $35,1 \pm 9,0$ & 0,423 \\
Idade do Cuidador & & & & \\
\hline
\end{tabular}

NSE: nível socioeconômico; DP: desvio padrão.

Tabela 2 - Comparação das habilidades funcionais e assistência do cuidador de crianças com paralisia cerebral leve, moderada e grave de classe econômica alta e baixa, descritos em média \pm desvio padrão

\begin{tabular}{|c|c|c|c|c|c|}
\hline \multirow{2}{*}{ Grupos } & & & \multicolumn{2}{|c|}{ Nível socioeconômico } & \multirow{2}{*}{ Valor $p$} \\
\hline & & & Alto & Baixo & \\
\hline \multirow{6}{*}{ Autocuidado } & \multirow{2}{*}{ PC leve } & $\mathrm{HF}$ & $51,2 \pm 12,2$ & $44,2 \pm 17,7$ & 0,376 \\
\hline & & $A C$ & $26,2 \pm 8,2$ & $21,4 \pm 13,3$ & 0,392 \\
\hline & \multirow{2}{*}{ PC moderado } & $\mathrm{HF}$ & $44,9 \pm 15,8$ & $36,2 \pm 17,95$ & 0,312 \\
\hline & & $A C$ & $23,4 \pm 7,7$ & $14,7 \pm 12,5$ & 0,124 \\
\hline & \multirow{2}{*}{ PC grave } & $\mathrm{HF}$ & $20,2 \pm 6,8$ & $8,1 \pm 11,3$ & 0,021 \\
\hline & & $A C$ & $4,4 \pm 4,9$ & $2,3 \pm 4,5$ & 0,407 \\
\hline \multirow{6}{*}{ Mobilidade } & \multirow{2}{*}{ PC leve } & $\mathrm{HF}$ & $44,1 \pm 6,6$ & $47,1 \pm 10,8$ & 0,516 \\
\hline & & $A C$ & $26,6 \pm 3,6$ & $27,2 \pm 5,3$ & 0,788 \\
\hline & \multirow{2}{*}{ PC moderado } & $\mathrm{HF}$ & $31,4 \pm 11,0$ & $25,3 \pm 11,2$ & 0,280 \\
\hline & & $A C$ & $22,1 \pm 5,7$ & $16,9 \pm 7,7$ & 0,149 \\
\hline & \multirow{2}{*}{ PC grave } & $\mathrm{HF}$ & $6,6 \pm 1,7$ & $2,91 \pm 2,6$ & 0,005 \\
\hline & & $A C$ & $2,0 \pm 1,9$ & $0,3 \pm 0,6$ & 0,014 \\
\hline \multirow{6}{*}{ Função social } & \multirow{2}{*}{ PC leve } & $\mathrm{HF}$ & $40,0 \pm 10,4$ & $32,5 \pm 16,0$ & 0,288 \\
\hline & & $A C$ & $20,7 \pm 3,4$ & $17,0 \pm 6,4$ & 0,167 \\
\hline & \multirow{2}{*}{ PC moderado } & $\mathrm{HF}$ & $42,9 \pm 13,1$ & $29,6 \pm 16,2$ & 0,094 \\
\hline & & $A C$ & $21,1 \pm 5,7$ & $11,4 \pm 9,3$ & 0,027 \\
\hline & \multirow{2}{*}{ PC grave } & $\mathrm{HF}$ & $22,4 \pm 19,5$ & $8,4 \pm 10,9$ & 0,194 \\
\hline & & $\mathrm{AC}$ & $10,8 \pm 10,4$ & $3,6 \pm 5,9$ & 0,098 \\
\hline
\end{tabular}

HF: Habilidades Funcionais; AC: assistência do cuidador. 
Para as habilidades funcionais do autocuidado $(p=0,021) \mathrm{e}$ mobilidade $(p=0,005)$ de crianças com PC grave, quando comparados os grupos de NSE alto e baixo, o desempenho daquelas de classe econômica baixa foi inferior nas habilidades de autocuidado e mobilidade. Ao verificar a independência na mobilidade, as crianças de NSE baixo recebem mais ajuda de seus cuidadores do que as de NSE alto ( $p=0,014)$; no entanto, não foi evidenciada diferença na independência do autocuidado $(p=0,407)$ e na função social $(p=0,098)$, indicando que, em ambos os grupos (NSE alto e baixo), as crianças necessitam de assistência equivalente.

A Tabela 3 mostra a comparação entre a classe econômica alta e baixa nas habilidades funcionais e na assistência do cuidador, independentemente da gravidade da PC. Em relação ao autocuidado, foi encontrada diferença na habilidade funcional $(0,027)$ e na independência $(0,032)$ das crianças, ou seja, os indivíduos de NSE baixo obtiveram desempenho inferior quando comparados àqueles de nível alto. $\mathrm{O}$ mesmo ocorreu na função social, na qual a participação das crianças de NSE baixo em atividades sociais foi menor do que entre aquelas de NSE alto. Entretanto, na mobilidade não foram encontradas diferenças significativas entre as duas classes sociais.

\section{Discussão}

De acordo com dados obtidos no presente estudo, o NSE de crianças com PC leve parece não afetar seu desempenho funcional. Buscando elucidar questões como essa, Andrade et a ${ }^{(21)}$ relataram que, no processo de reabilitação da criança, o NSE da família pode ser um obstáculo ou um facilitador, ou seja, nesse processo de aquisições de novas habilidades este pode ser um fator decisivo. Para Bracco et al ${ }^{(22)}$, o NSE baixo pode causar um comportamento inativo das crianças,

Tabela 3 - Comparação geral das habilidades funcionais e assistência do cuidador entre as classes econômicas alta e baixa

\begin{tabular}{|c|c|c|c|}
\hline \multirow{2}{*}{ Grupos } & \multicolumn{2}{|c|}{ Nível socioeconômico } & \multirow{2}{*}{ Valor $p$} \\
\hline & Alto & Baixo & \\
\hline \multicolumn{4}{|c|}{ Autocuidado* } \\
\hline $\mathrm{HF}$ & $41,2 \pm 17,5$ & $27,8 \pm 22,0$ & 0,027 \\
\hline $\mathrm{AC}$ & $19,8 \pm 11,6$ & $11,8 \pm 12,9$ & 0,032 \\
\hline \multicolumn{4}{|c|}{ Mobilidade } \\
\hline $\mathrm{HF}$ & $30,3 \pm 16,8$ & $22,8 \pm 20,0$ & 0,177 \\
\hline $\mathrm{AC}$ & $18,9 \pm 11,0$ & $13,4 \pm 12,4$ & 0,119 \\
\hline \multicolumn{4}{|c|}{ Função social } \\
\hline HF & $36,6 \pm 15,7$ & $22,4 \pm 17,7$ & 0,006 \\
\hline$A C$ & $18,4 \pm 7,6$ & $10,0 \pm 9,02$ & 0,001 \\
\hline
\end{tabular}

*média \pm desvio padrão; HF: habilidades funcionais; AC: assistência do cuidador. relacionado com alternativas restritas de lazer e cultura. Por outro lado, Malina e Bouchard ${ }^{(23)}$ verificaram que crianças com NSE baixo vivem em um ambiente com maior liberdade de movimentos, tendo mais oportunidades de vivenciar um repertório motor variado.

Quanto à função social dos indivíduos analisados, crianças com comprometimento variado (leve, moderado e grave) mostraram desempenho semelhante. Entretanto, quando se analisou a assistência do cuidador nessa mesma função, aquelas com PC moderada de NSE baixo se mostraram mais dependentes do que as crianças com NSE elevado, indicando que, embora apresentassem as habilidades necessárias, continuavam a receber ajuda de seus cuidadores. A socialização dessas crianças depende da família desempenhar o papel de mediadora entre a criança e a sociedade ${ }^{(24)}$, fornecendo suporte adequado, quando necessário, para que o indivíduo se integre ao grupo em que nasceu, adquirindo seus hábitos e valores característicos.

Famílias de distintos NSE podem ter expectativas diferentes acerca do desempenho independente das crianças com PC. Por isso, pode haver uma diferenciação na quantidade de auxílio que os cuidadores oferecem a essas crianças. O auxílio excessivo torna a criança mais dependente, mesmo que ela apresente condições de efetuar determinada tarefa. Buscando elucidar questões como essas, Bradley e Corwyn ${ }^{(25)}$ relataram que, no ambiente familiar, a criança pode receber assistência adequada para conviver com riscos para seu desenvolvimento. Nesse caso, o auxílio excessivo do cuidador poderia se tornar um risco para o desenvolvimento, além de uma sobrecarga ao cuidador. Camargos et a ${ }^{(26)}$,em um estudo com cuidadores de crianças com PC, verificaram uma maior sobrecarga entre aqueles que se encontravam em condições socioeconômicas desfavoráveis, ou seja, quanto menor o NSE, maior seria a sobrecarga do cuidador.

O risco social de pertencer a uma família de classe econômica desfavorecida parece afetar mais crianças com PC de comprometimento grave, ou seja, com maior risco biológico. Isso ocorreu principalmente na mobilidade, para a qual tanto a habilidade quanto a independência foram menores nas crianças com NSE baixo. Já no autocuidado, apesar de essas crianças terem habilidade inferior à das crianças de NSE alto, a assistência do cuidador foi semelhante em ambas as classes econômicas. Isso parece indicar que, quando se trata de crianças com comprometimento grave, famílias de classe econômica alta são mais superprotetoras do que aquelas de NSE baixo. O auxílio excessivo do cuidador pode ocorrer por diversos motivos; um deles é que o cuidador subestima a capacidade da criança para realizar atividades e acaba 
por fazer as tarefas, criando maior dependência. Shonkoff e Meisels ${ }^{(27)}$ alegam que cuidadores de crianças com sequelas mais graves, como é o caso da PC grave, exercem por um longo período papel dominante na interação cuidador e criança.

$\mathrm{Na}$ comparação geral do desempenho funcional, sem levar em consideração o comprometimento motor, observou-se que as famílias de classe econômica alta fornecem mais oportunidades para o desenvolvimento funcional do autocuidado do que as de classe econômica baixa. Tal dado corrobora o estudo realizado por Halpern et $a^{(7)}$, que constatou que crianças pertencentes a famílias de NSE baixo apresentaram duas vezes mais chances de atraso em seu desenvolvimento neuropsicomotor comparadas àquelas com NSE superior. Em um estudo feito por Dowding e Barry ${ }^{(28)}$, revelou-se que a classe social afetou os casos mais graves de PC.

Mancini et al ${ }^{(19)}$ mencionam que "o elevado NSE das famílias está relacionado a determinadas condições favoráveis, como maior escolaridade dos pais, maior acesso à informação e maior poder aquisitivo". Com o aumento dos recursos familiares, os pais tornam-se mais capazes de dar atenção e investir em seus filhos, seguindo as orientações de profissionais da saúde e da educação ${ }^{(29)}$. Por outro lado, pessoas com condições socioeconômicas desfavoráveis, expressas por meio do desemprego materno/ paterno e indisponibilidade dos bens de consumo, apresentam crianças com um desenvolvimento atrasado ${ }^{(30)}$. Devem-se considerar os diferentes contextos aos quais essas crianças estão expostas para padronizar a avaliação da funcionalidade, levando em consideração os fatores socioambientais, pois o NSE é o motivo ambiental mais relevante que pode contribuir para o processo de reabilitação ${ }^{(21)}$. Devido à natureza multifatorial e dinâmica do desenvolvimento infantil e da pobreza, a tentativa de confrontar dados de resultados de estudos que explicam a associação entre esses dois elementos é complexa ${ }^{(30)}$, sendo que a metodologia utilizada para avaliar tanto o NSE quanto o desenvolvimento infantil não é uniforme entre os diversos estudos ${ }^{(1,5,6,8,30-32)}$.

Os resultados apresentados contribuem para uma melhor compreensão do desenvolvimento das crianças com PC, sobretudo aquelas que se encontram em condições socioeconômicas diferenciadas. Entretanto, algumas limitações foram encontradas, uma vez que a amostra estudada foi reduzida e restrita a crianças com PC frequentadoras de programas de reabilitação em instituições especializadas. Além disso, houve a dificuldade de encontrar na região crianças com PC grave de NSE alto, resultando no reduzido número de integrantes desse grupo.

Sugere-se, a partir deste estudo, que o NSE possa exercer influência na gravidade do quadro e no desenvolvimento da criança com PC, devendo ser fator de risco considerado nas ações educacionais e de saúde voltadas a essa população. Estudos longitudinais poderiam contribuir com informações mais precisas, trazendo perspectivas de maior impacto nas políticas públicas, com propostas direcionadas aos setores de prevenção e reabilitação do Brasil.

\section{Agradecimentos}

À Coordenação de Aperfeiçoamento de Pessoal de Nível Superior (Capes), pela bolsa de Mestrado, e ao Fundo MackPesquisa, pelo apoio e reserva técnica.

\section{Referências bibliográficas}

1. Grantham-McGregor S, Cheung YB, Cueto S, Glewwe P, Richter L, Strupp B et al. Developmental potential in the first 5 years for children in developing countries. Lancet 2007;369:60-70.

2. Fleitlich BW, Goodman R. Epidemiologia. Rev Bras Psiquiatr 2000;22 (Suppl 2):2-6.

3. Halpern R, Figueiras AC. Environmental influences on child mental health. J Pediatr (Rio J) 2004;80 (Suppl 2):S104-10.

4. Gabbard CP. Lifelong motor development. $5^{\text {th }}$ ed. Redwood City: Benjamin Cummings; 2008.

5. Barros KM, Fragoso AG, de Oliveira AL, Cabral Filho JE, de Castro RM. Do environmental influences alter motor abilities acquisition? A comparison among children from day-care centers and private schools. Arq Neuropsiquiatr 2003;61:170-5.
6. Lima MC, Eickmann SH, Lima AC, Guerra MQ, Lira PI, Huttly SR et al. Determinants of mental and motor development at 12 months in a low income population: a cohort study in northeast Brazil. Acta Paediatr 2004;93:969-75.

7. Halpern R, Giugliani ER, Victora CG, Barros FC, Horta BL. Risk factors for suspicion of developmental delays at 12 months of age. J Pediatr (Rio J) 2000;76:421-8.

8. Andraca I, Pino P, de La Parra A, Rivera F, Castillo M. Risk factors for psychomotor development among infants born under optimal biological conditions. Rev Saude Publica 1998;32:138-47.

9. Victora CG, Huttly SR, Barros FC, Lombardi C, Vaughan JP. Maternal education in relation to early and late child health outcomes: findings from a Brazilian cohort study. Soc Sci Med 1992;34:899-905. 
10. Schenker R, Coster WJ, Parush S. Neuroimpairments, activity performance, and participation in children with cerebral palsy mainstreamed in elementary schools. Dev Med Child Neurol 2005;47:808-14.

11. Nelson CA. Paralisia cerebral. In: Umphred DA, editor. Reabilitação neurológica. $4^{\text {th }}$ ed. São Paulo: Manole; 2004.

12. Finnie NR. O manuseio em casa da criança com paralisia cerebral. $3^{\text {rd }}$ ed. São Paulo: Manole; 2001.

13. Sundrum R, Logan S, Wallace A, Spencer N. Cerebral palsy and socioeconomic status: a retrospective cohort study. Arch Dis Child 2005;90:15-8.

14. Palisano RJ, Hanna SE, Rosenbaum PL, Russell DJ, Walter SD, Wood EP et al. Validation of a model of gross motor function for children with cerebral palsy. Phys Ther 2000;80:974-85.

15. Palisano R, Rosenbaum P, Bartlett D, Livingston M. Gross motor function classification system: expanded and revised. CanChild Centre for Childhood Disability Research [homepage on the Internet]. 2007 [cited 2009 Mar 4]. Available from: http://motorgrowth.canchild.ca/en/GMFCS/resources/GMFCS-ER.pdf

16. Palisano R, Rosenbaum P, Walter S, Russell D, Wood E, Galuppi B. Development and reliability of a system to classify gross motor function in children with cerebral palsy. Dev Med Child Neurol 1997;39:214-23.

17. Mancini MC, Alves AC, Shaper C, Figueiredo EM, Sampaio RF, Coelho ZA et al. Gravidade da paralisia cerebral e desempenho funcional. Rev Bras Fisioter 2004;8:253-60.

18. Associação Brasileira de Empresas de Pesquisa [homepage on the Internet]. Critério de classificação econômica Brasil. [cited 2012 Sept 14]. Available from: www.abep.org/novo/FileGenerate.ashx?id=252

19. Mancini MC, Megale L, Brandão MB, Melo AP, Sampaio RF. The moderating effect of social risk in the relationship between biologic risk and child functional performance. Rev Bras Saude Mater Infant 2004;4:25-34.

20. Mancini MC. Inventário de avaliação pediátrica de incapacidade (Pedi): manual da versão brasileira adaptada. Belo Horizonte: Universidade Federal de Minas Gerais; 2005.
21. Andrade PM, Ferreira FO, Vasconcelos AG, Lima EP, Haase VG. Cognitive profile, motor deficits and influence of facilitators for rehabilitation of children with neurological dysfunction. Rev Paul Pediatr 2011;29:320-7.

22. Bracco MM, Colugnati FA, Pratt M, Taddei JA. Multivariate hierarchical model for physical inactivity among public school children. J Pediatr (Rio J) 2006;82:302-7.

23. Malina RM, Bouchard C. Growth of latin american children:socio-economic urban-rural and secular comparisons. Rev Bras Cienc Mov 1990;4:46-72.

24. Andrade SA, Santos DN, Bastos AC, Pedromônico MR, de Almeida-Filho N, Barreto $\mathrm{ML}$ et al. Family environment and child's cognitive development: an epidemiological approach. Rev Saude Publica 2005;39:606-11.

25. Bradley RH, Corwyn RF. Socioeconomic status and child development. Ann Rev Psychol 2002;53:371-99.

26. Camargos AC, Lacerda TT, Viana SO, Pinto LR, Fonseca ML. Assessment of the caregiver burden of children with cerebral palsy using the Burden interview scale. Rev Bras Saude Mater Infant 2009;9:31-7.

27. Shonkoff JP, Meisels SJ. Handbook of early childhood intervention. New York: Cambridge University; 1990.

28. Dowding VM, Barry C. Cerebral Palsy: social class differences in prevalence in relation to birthweight and severity of disability. J Epidemiol Community Health 1990;44:191-5.

29. Schreiber J, Effgen SK, Palisano RJ. Effectiveness of parental collaboration on compliance with a home program. Pediatr Phys Ther 1995;7:59-64.

30. Paiva GS, Lima AC, Lima MC, Eickmann SH. The effect of poverty on developmental screening scores among infants. Sao Paulo Med J 2010;128:276-83.

31. Miller JE. Developmental screening scores among preschool-aged children: the roles of poverty and child health. J Urban Health 1998;75:135-52.

32. Evans GW, Kim P. Childhood poverty and health: cumulative risk exposure and stress dysregulation. Psychol Sci 2007;18:953-7. 